\title{
I sogni di Zeno
}

\section{Giovanni Albertocchi}

Universitat de Girona

\section{Abstract}

Nel redigere il proprio diario, come gli ha consigliato il suo analista, Zeno Cosini non tralascia di annotare una decina di sogni fatti in diverse circostanze. Nel presente articolo si cerca di analizzare la tipologia di questi sogni, differenziando quelli costruiti intenzionalmente per farsi beffe dell'analista, da quelli che sembrano rispettare l'ortodossia freudiana.

Parole chiave: analista, sogno, inconscio, desiderio, morte del padre.

\section{Abstract}

Composing his own diary as suggested by his analyst, Zeno Cosini writes about the his dreams in different situations. In this article we analyse the typology of these dreams, distinguishing between those intentionally constructed to mock the analyst and those that seem to respect Freudian orthodoxy.

Key words: psychoanalyst, dream, unconscious, desire, father's death.

Nella finzione voluta dall'autore, La coscienza di Zeno, sarebbe un diario in cui il protagonista annota, su indicazione del suo psico-analista, il Dottor S., i suoi ricordi e le sue riflessioni. Nel descrivere la sua vita, Zeno non tralascia di appuntare alcuni sogni, una decina, fatti in epoche diverse, che danno a noi lettori la possibilità di curiosare nel suo inconscio. Anche se si tratta, è bene precisare, di un inconscio artefatto: in primo luogo perché abbiamo a che fare con un personaggio di finzione che sogna con i materiali che gli mette a disposizione il suo autore e poi perché tale personaggio è abituato a mentire, come ha fatto notare il Dottor S. nella «Prefazione».

Il percorso che compie il lettore all'interno del romanzo si svolge in effetti all'insegna dell'insicurezza e dell'ambiguità: per usare le stesse parole del personaggio, il lettore non fa che chiedersi se Zeno sia "buono o cattivo", se sta dicendo la verità o se sta mentendo spudoratamente. Poi alla fine non resta 
che accettare questo singolare patto narrativo che ci spiazza in continuazione ed ammettere, come suggerisce l'autore, che tale ambiguità possa essere in fondo un patrimonio da salvaguardare.

Come afferma Jean Pouillon, Zeno mente per essere capito: i dubbi sulla sua presunta malafede, sono varianti elargite consapevolmente per aiutarci a scoprire la verità. ${ }^{1}$

Ma torniamo ai sogni. Ve ne sono di due tipi: quelli «autentici», si fa per dire, cioè fatti dal protagonista nella circostanza «ufficiale» del sonno e quelli che lui costruisce appositamente per ingannare l'analista. Tutti quelli, ad esempio, che appaiono nell'ultimo capitolo, "Psico-analisi», potrebbero essere stati intenzionalmente manipolati dallo spirito polemico del personaggio nei confronti della terapia.

Dopo che il dottor S. lo ha avvertito che è afflitto dalla malattia "diagnosticata a suo tempo dal defunto Sofocle sul povero Edipo", e che, per farla breve «avevo amata mia madre e avrei voluto ammazzare mio padre», ${ }^{2}$ Zeno insorge contro l'analista e l'intera psico-analisi, a suo dire soltanto «un trucco buono per commovere qualche vecchia donna isterica». ${ }^{3}$ Sferra l'attacco finale con una sequenza di cinque sogni inventati a bella posta per farsi beffe dell'analista. Cinque scene in cui si prende gioco del «dramma» edipico che gli è stato affibbiato con i relativi protagonisti.

Così in uno dei sogni, troviamo Zeno bambino divorato dalla gelosia per il fratellino minore che se ne sta a casa con la mamma mentre lui è "condannato ad andare sempre a scuola». ${ }^{4}$ Per la cronaca, scoprirà in seguito che il fratello era invidioso di lui esattamente per il contrario, perché doveva andare a scuola e lasciare il rivale solo, a casa, con la mamma. C'è poi il sogno relativo alla gelosia per il padre che gli usurpa l'oggetto del desiderio. La scena è molto divertente: il Sig. Cosini, tornato a casa dal lavoro, si gode la confortevole quiete famigliare, accanto alla moglie che disbriga le faccende domestiche. Per l'esattezza sta scrivendo con dell'inchiostro indelebile le iniziali sulla biancheria di qualche membro della famiglia. Zeno, che gioca sotto il tavolo, manda tutto all'aria, provocando l'ira del padre che cerca di sferrargli un calcio:

Il nostro tinello nelle ore pomeridiane. Mio padre è ritornato a casa e siede su un sofa accanto alla mamma che sta imprimendo con certo inchiostro indelebile delle iniziali su molta biancheria distribuita sul tavolo a cui essa siede. Io mi trovo sotto il tavolo dove giuoco con delle pallotole. M'avvicino sempre più a mamma. Probabilmente desidero ch'essa s'associ ai miei giuochi. A un dato punto, per rizzarmi in piedi fra di loro, m'aggrappo alla biancheria che pende dal tavolo e allora avviene un disastro. La boccetta d'inchiostro mi capi-

1. Jean POUILlon, "La conscience de Zeno: roman d'un psychanalyse ", Les temps modernes, n. 106, 1954, vol. X, p. 555-562.

2. Italo Svevo, La coscienza di Zeno, in Romanzi, a cura di Mario Lavagetto, Torino: Einaudi-Gallimard, 1993, p. 865.

3. Ibid., p. 877.

4. Ibid., p. 867. 
ta sulla testa, bagna la mia faccia e le mie vesti, la gonna di mamma e produce una lieve macchia anche sui calzoni di papà. Mio padre alza una gamba per appiopparmi un calcio... 5

Senonché, per sua fortuna, Zeno si sveglia proprio mentre il padre sta per sferrargli un calcio, e si trova così in salvo: «Ma io in tempo ero ritornato dal mio lontano viaggio e mi trovavo al sicuro qui, adulto, vecchio». ${ }^{6}$

Negli altri due sogni, sempre appartenenti all'ultimo capitolo del romanzo, troviamo ancora Zeno bambino che osserva una donna formosa, con un provocante vestito nero e delle scarpine di lacca, chiusa in una gabbia. Il bambino la osserva estasiato e goloso, immaginando di assaggiarla come se fosse un dolce prelibato:

[...] una donna formosa, costruita deliziosamente, vestita di nero, bionda, dagli occhi grandi e azzurri, le mani bianchissime e i piedi piccoli in scarpine laccate delle quali, di sotto alle gonne, sporgeva solo un lieve bagliore. Tutto era lei! Ed il bambino sognava di possedere quella donna, ma nel modo più strano: era sicuro cioè di poter mangiarne dei pezzetini al vertice e alla base. $^{7}$

Il sogno suscita naturalmente l'interesse dello psico-analista che assapora la conferma della diagnosi edipica. Di fronte alla sua impazienza, Zeno, in una seduta successiva, accelera la diagnosi, inventandosi di sana pianta il finale tanto desiderato dal Dottor S. Infatti «vediamo» il bambino che si avvicina alla gabbia e prega la donna dal vestito nero di porgergli un piedino «da succhiare e da mangiare». Il «sinistro», precisa implacabile Zeno mentre racconta il sogno all'analista che è convinto di veder confermata la teoria. "Dimostravo così - precisa Zeno - di aver capito perfettamente la malattia che il dottore esigeva da me. Edipo infantile era fatto proprio così: succhiava il piede sinistro della madre per lasciare il destro al padre». ${ }^{8}$

Prescindendo dall' ultimo capitolo del romanzo che offre, come si è appena visto, sequenze oniriche strumentali alla polemica ed alla satira, troviamo nel corso dell'opera sogni ben più interessanti.

Il primo che vorrei analizzare, si svolge all'insegna della più rigida ortodossia freudiana. Lo conferma lo stesso autore in uno scritto autobiografico, "Soggiorno londinese», allorché, come succede spesso, civetta con la sua presunta liaison con la psico-analisi di Sigmund Freud. Sembra quasi che nei riguardi dello scienziato viennese, Svevo sia afflitto dagli stessi sintomi di ambivalenza che traspaiono nel comportamento di Zeno nei confronti di chi lo circonda.. Comunque, nello scritto appena citato si legge che nel romanzo «Vi sono due o tre idee [...] prese di peso dal Freud. L'uomo che per non assiste-

5. Ibid., p. 869.

6. Ibid.

7. Ibid., p. 870 .

8. Ibid., p. 871-872. 
re al funerale di colui che diceva suo amico e ch'era in realtà suo nemico si sbaglia di funerale è Freudiana con un coraggio di cui mi vanto. ${ }^{9}$ L'altro che sogna di avvenimenti lontani e nel sogno li altera come avrebbe voluto fossero stati, è Freudiano in modo come saprebbe fare chiunque conosca il Freud». ${ }^{10}$

Più avanti lo scrittore precisa di aver apprezzato di Freud, per ragioni di chiarezza, soprattutto le «sue celebri prelezioni che conobbi appena nel 1916». ${ }^{11}$

Il critico Mario Lavagetto le identifica con le cinque conferenze Sulla psicanalisi tenute da Freud nel 1909 alla Clark University di Worcester, tradotte in italiano da Marco Levi Bianchini nel 1915 e che avrebbero potuto quindi essere la fonte delle «due o tre idee» di cui parla Svevo. ${ }^{12}$ Si tratta di un testo assai chiaro, didattico, ove, si delineano i due principi notissimi, la compensazione e lo spostamento, che governano l'attività onirica e che ritroviamo, applicati diligentemente da Svevo, nel famoso «sogno delle mignatte».

Ci troviamo nell'episodio della morte del padre: il povero Zeno è alle prese con la malattia del congiunto che ha ormai le ore contate. Il medico curante, il dottor Coprosich, per farlo tornare in sé vorrebbe applicare all'infermo le sanguisughe e parla anche della possibilità di far ricorso alla camicia di forza, nel caso ce ne fosse bisogno. Zeno reagisce energicamente e si oppone a quella che considera una inutile crudeltà.

Allora m’arrabiai. Poteva esserci un'azione più malvagia di quella di richiamare in sé un ammalato, senz'avere la minima speranza di salvarlo e solo per esporlo alla disperazione, o al rischio di dover sopportare — con quell'affanno! - la camicia di forza? Con tutta violenza, ma sempre accompagnando le mie parole di quel pianto che domandava indulgenza, dichiarai che mi pareva una crudeltà inaudita di non lasciar morire in pace chi era definitivamente condannato. ${ }^{13}$

Il gesto pietoso nei confronti del padre, manipolato dall'orgoglio ferito del medico che gli rimprovera di voler «recidere anche quel tenue filo di speranza», ${ }^{14}$ si trasforma in uno dei diversi ingredienti del senso di colpa che di lì a poco, si abbatterà, con la precisione di uno schiaffo, sulla guancia del giovane.

Si tratta di una delle scene più note del romanzo. Zeno accorre al capezzale del padre che, ormai agonizzante, cerca confusamente di alzarsi dal letto. Il dottore aveva raccomandato il riposo assoluto, per cui Zeno cerca di tenerlo fermo, immobilizzandolo; ma il vecchio, in un ultimo sforzo, si solleva in

9. Si riferisce all'episodio in cui Zeno, che sta seguendo il funerale del cognato Guido Speier, perde di vista il corteo funebre e si ritrova in un cimitero ortodosso. Bisogna precisare che Zeno non ha mai avuto eccessiva simpatia per Guido che gli ha sottratto Ada, la donna di cui si era innamorato.

10. Italo Svevo, Teatro e Saggi, Federico Bertoni (a cura di), Milano: Arnoldo Mondadori Editore, 2004, p. 894.

11. Ibid., p. 897.

12. Mario LaVAgETTO, L'impiegato Schmitz e altri saggi su Svevo, Torino: Einaudi, 1986, p. 42.

13. Italo SVEVo, La coscienza di Zeno, p. 550-551.

14. Ibid., p. 551. 
piedi sul letto per poi ricadere fulminato. Accasciandosi ormai privo di vita, colpisce con la mano la guancia del figlio: "Con uno sforzo supremo arrivò a mettersi in piedi, alzò la mano alto alto, come se avesse saputo ch'egli non poteva comunicarle altra forza che quella del suo peso e la lasciò cadere sulla mia guancia. Poi scivolò sul letto e di là sul pavimento. Morto!». ${ }^{15}$

Non sapremo mai esattamente se l'ultimo gesto del padre sia stato un movimento convulso provocato dall'agonia oppure un colpo preciso, uno schiaffo, al figlio che gli impedisce di muoversi. Comunque, se ci atteniamo al punto di vista di Zeno, è logico che in quelle circostanze lo sentisse come una punizione.

Il rifiuto delle mignatte e della camicia di forza ha prodotto infatti come effetto secondario il rimorso per aver desiderato di accelerare (o aver desiderato tout court) la morte del padre, da qui il castigo terribile che il figlio sente di aver meritato.

Anni dopo, esattamente nell'epoca in cui scrive le proprie memorie, quell'episodio lontano subisce un salutare ribaltamento. Zeno sogna di essere lui questa volta ad imporre energicamente, battendo il pugno su un libro di medicina, le sanguisughe e la camicia di forza al padre malato, mentre il ruolo umanitario e di riflesso anche la colpa, è ceduto al dottor Coprosich.

Mi rivedevo col dottore nella stessa stanza ove avevamo discusso di mignatte e camicie di forza, in quella stanza che ora ha tutt'altro aspetto perché è la stanza da letto mia e di mia moglie. Io insegnavo al dottore il modo di curare e guarire mio padre, mentre lui (non vecchio e cadente com'è ora, ma vigoroso e nervoso com'era allora) con ira, gli occhiali in mano e gli occhi disorientati, urlava che non valeva la pena di fare tante cose. Diceva proprio così: «Le mignatte lo richiamerebbero alla vita e al dolore e non bisogna applicargliele!» Io invece battevo il pugno su un libro di medicina e urlavo: «Le mignatte! Voglio le mignatte! Ed anche la camicia di forza!» ${ }^{16}$

Il sogno, con una perfetta simmetria risarcisce quell'antico dolore di Zeno, capovolgendo i ruoli e le relative responsabilità dei protagonisti. Una sorta di aiuto che gli offre l'inconscio per cercare di ricondurre il gesto finale del padre nell'ambito di una tragica fatalità e non in quello di un terribile castigo.

Lo scambio dei ruoli fra Zeno e Coprosich avviene con la precisione di un fenomeno scientifico, come ricorda il nostro personaggio che da vecchio dice di osservare e capovolgere quelle ombre lontane con «un ausilio ottico»: "Ombre lontane! Io credo che per scorgervi occorra un ausilio ottico e sia questo che vi capovolga». ${ }^{17}$ Lidea di osservare o di fissare il proprio passato attraverso una lente che rovescia le immagini, è rivendicata - lo abbiamo già visto- nel «Soggiorno londinese» come unico contributo originale alla elaborazione di un sogno che, come dice Svevo, «saprebbe fare chiunque conosca il Freud». 
L'autore precisa infatti che non avrebbe nessuna ragione di andarne orgoglioso, «se non vi fosse dentro un'altra ideuccia di cui mi compiaccio».

L'episodio della morte del padre, con la sua complessa trama di ripercussioni, mi ha fatto ripensare ad un'analoga e drammatica esperienza vissuta personalmente dallo scrittore nordamericano Philip Roth al capezzale del padre e che viene descritta in un'opera affascinante, Patrimony. A true story, apparsa nel 1991. ${ }^{18}$ Nel titolo abbiamo già il valore che assumerà tale esperienza per lo scrittore americano: l'assistere il padre durante la malattia fino al decesso, sarà infatti il suo patrimonio esistenziale e non un dramma con il finale tragi-comico del ceffone. Vi è comunque un nesso fra i due personaggi che subiscono ritorsioni analoghe da parte dell'inconscio che agisce evidentemente, al di là delle diversità culturali e dell'epoca, in base a leggi comuni.

Philip e Zeno sperimentano di fronte alla malattia del genitore la sensazione di trovarsi spiazzati nei confronti del principio che sembra fissare il rapporto padre-figlio sui ruoli rispettivi di forte e di debole. Nel caso di Zeno, la rottura di tale schema raggiunge l'apice nel momento finale quando è costretto ad usare tutta la sua forza per trattenere il vecchio che vorrebbe alzarsi dal letto. La trasgressione di quel principio ancestrale produce istantaneamente il terribile castigo che tutti conosciamo e che lascia il povero Zeno in balia di insanabili rimorsi.

Poi, con il passare del tempo, un sogno ad occhi aperti viene finalmente a risarcire quella ferita, ricomponendo l'equilibrio di forza e debolezza stravolto dalle circostanze. «Divenni buono, buono - racconta Zeno- e il ricordo di mio padre s'accompagnò a me, divenendo sempre più dolce. Fu come un sogno delizioso: eravamo ormai perfettamente d'accordo, io divenuto il più debole e lui il più forte». ${ }^{19}$

Per Philip Roth, la trasgressione passa anche attraverso la scrittura che consolida la posizione di forza acquisita assistendo il padre e descrivendone per di più la malattia, con le sue inevitabili brutture, e la morte. Nonostante lo splendido rapporto che hanno i due (il padre reale, va detto, è assai lontano dall'ossessivo padre-personaggio che appare nella saga narrativa di Portnoy ${ }^{20}$ e di Zuckerman), ${ }^{21}$ c'è qualcosa che stona in quel finale e che spinge evidentemente Philip Roth ad esigere anche lui la sua quota di risarcimento. E lo ottiene alla fine allorché gli appare in sogno il padre che lo rimprovera per non averlo seppellito con il vestito adeguato. Per una forma di zelo ebraico lo avevano avvolto semplicemente in un sudario. Il rimprovero cade come una manna sull'inconscio dello scrittore che recupera l'immagine «ufficiale» di un padre forte nell'atto di giudicare il figlio e di sanzionarne la condotta. Lasciamo che sia Philip Roth a raccontare la scena:

18. Philip Roth, Patrimony. A true story, New York: Vintage Books, Random House Inc., 1991.

19. Ibid., p. 559.

20. È il protagonista di Portnoy's Complaint (1967).

21. Mi riferisco ai diversi romanzi di cui è protagonista Nathan Zuckerman: The Gost Writer (1979), Zuckerman Unbound (1981), The Counterlife (1986), ecc. 
Mi svegliai urlando. Tutto ciò che faceva capolino dal sudario era il rammarico sulla sua faccia morta. E le uniche parole che disse erano una ramanzina: l'avevo vestito per l'eternità con i panni sbagliati.

$\mathrm{Al}$ mattino mi resi conto che aveva inteso alludere a questo libro, che, in carattere con l'indecenza della mia professione, avevo continuato a scrivere mentre lui era malato e moriva. Il sogno mi diceva che, se non nei miei libri o nella mia vita, almeno nei miei sogni sarei vissuto in eterno come il suo figlio piccolo, con la coscienza di un figlio piccolo, proprio come lui sarebbe rimasto vivo non soltanto come mio padre ma come $i l$ padre, per giudicarmi qualunque cosa io faccia.

Non devi dimenticare nulla. ${ }^{22}$

Altro sogno assai noto della Coscienza di Zeno è il «sogno di Basedow». È quello che dispone anche della bibliografia più consistente, basti citare $\mathrm{i}$ nomi di Eduardo Saccone e di Mario Lavagetto che sono tra i critici più prestigiosi dell'opera di Italo Svevo. Ed è anche quello più difficile da interpretare, costruito, come ha rilevato il Lavagetto, su una specie di «ombelico» indecifrabile. Eccone la trama.

Vi troviamo in azione il solito "trio» di personaggi che il destino e la «malattia» di Zeno hanno ormai indissolubilmente legato, ossia Augusta (la moglie), Ada (la cognata) e Zeno, affacciati, in quest'ordine, ad una finestra che da' sul Corso. Ada è al centro e pare appoggiarsi volutamente a Zeno, sempre sensibile al fascino della cognata, anche se appannatto da quella strana malattia (il morbo di Basedow) che le altera le fattezze armoniose. Zeno infatti si sorprende del fatto che Ada sia tornata desiderabile come era prima e si chiede dove sia finito Basedow. Augusta glielo indica con la mano: è un vecchio barbone che cerca di dileguarsi, giù in strada, inseguito dalla folla minacciosa che ripete la nota imprecazione manzoniana di «Ammazzate l'untore!».

Ecco il sogno: eravamo in tre, Augusta, Ada ed io che ci eravamo affacciati ad una finestra e precisamente alla più piccola che ci fosse stata nelle nostre tre abitazioni, cioè la mia, quella di mia suocera e quella di Ada. [...] Al piccolo davanzale c'era tanto poco spazio che Ada, che stava in mezzo a noi tenendosi alle nostre braccia, aderiva proprio a me. Io la guardai e vidi che il suo occhio era ridivenuto freddo e preciso e le linee della sua faccia purissime fino alla nuca ch'io vedevo coperta dei suoi riccioli lievi, quei riccioli ch'io avevo visti tanto spesso quando Ada mi volgeva le spalle. Ad onta di tanta freddezza (tale mi pareva la sua salute) essa rimaneva aderente a me come avevo creduto lo fosse quella sera del mio fidanzamento intorno al tavolino parlante. Io, giocondamente, dissi ad Augusta [...]: «Vedi com’è risanata? Ma dov'è Basedow?» «Non vedi?» domandò Augusta ch'era la sola fra di noi che arrivasse a guardare sulla via. Con uno sforzo ci sporgemmo anche noi e scorgemmo una grande folla che s'avanzava minacciosa urlando. "Ma dov'è Basedow?» domandai ancora una volta. Poi lo vidi. Era lui che s'avanzava inseguito da quella folla: un vecchio pezzente coperto di un grande mantello stracciato, ma di broccato 
rigido, la grande testa coperta di una chioma bianca disordinata, svolazzante all'aria, gli occhi sporgenti dall'orbita che guardavano ansiosi con uno sguardo ch'io avevo notato in bestie inseguite, di paura e di minaccia. E la folla urlava: «Ammazzate l'untore! ${ }^{23}$

Il sogno prosegue poi, dopo una breve pausa di veglia, in una seconda sequenza, dove il desiderio di Zeno per la cognata riprende in pieno il sopravvento. I due si stanno inerpicando, Ada sopra e Zeno sotto, su una scala che conduce ad una soffitta dove tutto lascia presagire che consumeranno l'atto sessuale. Ma d'improvviso Basedow fa capolino in cima alle scale e manda all'aria i loro piani. Zeno atterrito si da' alla fuga e si sveglia. Racconta trafelato il sogno ad Augusta che ne intuisce evidentemente il significato: il risultato è che la mattina dopo, sul viso di lei, appare «il cereo pallore delle grandi occasioni». Zeno cerca di difendersi, adducendo che Ada non c'entra, ma risulta poco credibile. Come confessa lui stesso, "quando si viene colti nel sogno è difficile di difendersi». ${ }^{24}$

Il pallore di Augusta ci fornisce la chiave più probabile del sogno che è quella del desiderio di Zeno per la cognata che costituisce un percorso narrativo di una certa consistenza, fatto di rimandi e di ricordi che possiamo considerare, alla stregua di Saccone, come momenti sostitutivi delle libere associazioni, a cui non abbiamo accesso, essendo, ripetiamo, l'inconscio di Zeno pura finzione narrativa.

Torniamo alla scena della finestra: il contatto con il corpo di Ada produce una associazione genuina di Zeno con un altro contatto altrettanto eccitante avvenuto tempo addietro: "[...] essa rimaneva aderente a me come avevo creduto lo fosse quella sera del mio fidanzamento intorno al tavolino parlante». Si riferisce all'episodio della seduta spiritica appartenente al capitolo «La storia del mio matrimonio»: Zeno, nell'oscurità, dichiara il suo amore alla donna sbagliata, Augusta, che scambia per Ada, di cui era innamorato. Il buio quella sera gli aveva giocato un brutto scherzo, perché in realtà la donna che sopportava in silenzio le sue avances era Augusta. A Zeno sarebbe toccato come «a quel tiratore - sono parole sue - cui era riuscito di colpire il centro del bersaglio, però di quello posto accanto al suo». ${ }^{25}$

Ora invece sembra che nel sogno abbia aggiustato la mira: Ada non è solo l'obbiettivo centrato, ma è addirittura lei a prendere l'iniziativa. Ne consegue quindi un doppio capovolgimento della scena reale. Come se l'inconscio di Zeno, per osservare le ombre del passato, avesse inforcato un'altra volta quel non meglio identificato artilugio ottico che capovolge le immagini. Anche qui, come nel sogno delle mignatte, i valori freudiani di compensazione e di spostamento sembrano affatto rispettati.

Per comprendere pienamente questo ritorno di fiamma di Zeno per la cognata, dobbiamo risalire a qualche pagina addietro: ancora una volta è in 
azione il solito trio. Sembra quasi che la presenza di Augusta favorisca, anziché frenarli, i cattivi pensieri del marito. Dunque, troviamo Ada, già provata dalla malattia, che si appoggia alla sorella ed al cognato, turbato da quell'innocente contatto che rimette subito in moto le antiche e mai sopite speranze. Stavolta però le circostanze paiono diverse, perché la malattia sembra offrire a Zeno nuove chance come conferma questa sua curiosa elucubrazione: «Non poteva la sua perversione portarla ad amare me, che da sana aveva respinto?». ${ }^{26}$ Per una di quelle inversioni così frequenti nel romanzo, Ada, imbruttita, è divenuta, per Zeno, ancor più seducente e desiderabile, in quanto ora è più accessibile di prima. Per non dare nell'occhio, Zeno la mette sul piano di una semplice transazione commerciale: certo che l'oggetto in questione ha perso di valore ma lui, ci tiene a precisare, è un gentiluomo che deve comunque mantenere fede al contratto.

Pochi anni prima io le avevo dichiarato il mio amore e non avevo fatto alcun atto di revoca fuori di quello di sposarne la sorella. In tale contratto essa non era protetta dalla legge ma dalla cavalleria. A me pareva di essere tanto fortemente impegnato con lei, che se essa si fosse presentata da me molti ma molti anni piú tardi, perfezionata magari nella malattia da Basedow da un bel gozzo, io avrei dovuto far onore alla mia firma. ${ }^{27}$

La malattia ha trasformato Ada in un ossimoro che si regge sui valori contrastanti di salute e di malattia a cui si affiancano quelli di bellezza e di bruttezza, ma non in forma direttamente proporzionale come vorrebbe la tradizione. Qui i valori sono spaiati (salute:bruttezza= malattia:bellezza), al punto di dover ammettere che Basedow ha "perfezionato", pur danneggiandola, la bellezza di Ada. Certo che per il desiderio di Zeno non è facile districarsi fra tutti questi equilibri in perenne movimento, più propri di un contabile che di un innamorato. Da qui il carattere intermittente che ha il desiderio nel sogno: prima viene autorizzato dalla fuga di Basedow che se ne va dopo aver compiuto la sua missione (quella di rimettere Ada sul mercato) e poi censurato, sul più bello, dalla riapparizione del medico che lo fa rientrare nei ranghi.

Secondo Eduardo Saccone, Basedow, la cui chioma bianca ricorda quella del padre di Zeno, ha la funzione castratrice nei riguardi di un eros che non fa che riprodurre il modello edipico. Ne consegue, sempre secondo il critico, che il "desiderio inconscio di Zeno non è[...] di fare l'amore con Ada, ma piuttosto che il suo desiderio di fare all'amore con Ada sia frustrato». ${ }^{28}$ Mario Lavaggetto invece preferisce fermarsi al di fuori di quella crosta impenetrabile da lui definita come ombelico. ${ }^{29}$

Per quanto mi riguarda, preferirei ricondurre Basedow e le sue competenze in quell'ambito preciso della trama, individuato dal dottor $S$, dove si anni- 
dano le menzogne del paziente. Si tratta, nonostante le riserve dell'analista, di una zona perfettamente legittimata dalle leggi narrative. Come afferma Zeno, per difendersi dalla ramanzina del dottor $S$., «inventare è una creazione, non già una menzogna». ${ }^{30} \mathrm{E}$ in fondo, potremmo aggiungere sulla scia della teoria già citata di Jean Pouillon ("Zeno mente per essere capito») un atto di generosità verso il lettore a cui si forniscono tutte le varianti possibili (non importa che siano vere o false) per capire il personaggio ed il suo percorso narrativo.

$\mathrm{Vi}$ sono nel romanzo, due livelli di invenzione: quella ufficiale che scorre con la sua brava funzione diegetica lungo la trama e quella segreta ove agisce un io che si confessa all'analista e che cerca di ingannarlo cambiando sovente le carte in tavola. L'inattendibilità è in fondo il rischio di qualsiasi confessione, sia fatta per iscritto che a viva voce. Da questa zona segreta proviene la famosa censura, smascherata dal dottor S., sul magazzino di legnami di Guido Speer che avrebbe potuto modificare, in parte, l'immagine di fallito che invece Zeno ci tiene ad offrire del cognato. Fortunatamente per noi, il dottor S. si improvvisa detective e scopre come stanno le cose.

Immaginiamo che anche nel confronti di Basedow, Zeno si comporti nello stesso modo. Cioè che all'interno della storia ufficiale che Zeno dovrebbe scrivere nel suo diario, Ada non si sia mai ammalata ed abbia quindi conservato intatto il suo fascino fino alla partenza per l'Argentina. Basedow non era previsto: è una variante supplementare che Zeno si inventa per legittimare, rendendolo più verosimile, il desiderio per la cognata. Il desiderio viene in un certo senso prorogato, in base a quel contratto che Zeno dice di non aver mai disdetto. È legittimato nella sua persistenza e consolidato dalla possibilità, questa volta, di essere soddisfatto: basta solo ritoccare l'immagine di Ada, rendendola più brutta e meno orgogliosa. Con il viso deformato in quel modo, stavolta non potrà rifiutare le avance del cognato. 